\title{
Analysis and Design of Public Sports Information Processing System
}

\author{
ZHOU Li \\ Physical Education ,Jiangxi Science and Technology Normal University,Nanchang ,China \\ a zhoulijx@163.com
}

Keywords: B/S structure, Access database, Public sports, Information Processing

\begin{abstract}
The construction and development of public sports has made unprecedented progress. It has played an important role in promoting the coordinated development of mass sports, competitive sports and sports industry. However, the current public sports service still exists the backwardness of management system, service ability is not strong, lack of integration of resources and other issues, and cannot meet the growing diversification, multi-level sports demand. According to the information processing system of public sports carried out preliminary analysis, it is proposed the system design based on Access database and B/S model technology, the majority of sports meet the needs of users, to promote the modernization of sports information and provide a theoretical basis for the.
\end{abstract}

\section{Introduction}

With the improvement of human social productive capacity, the change of lifestyle, the increase of leisure time, the decrease of physical labor, the decrease of people's basic activities and the appearance of all kinds of physical problems. Physical exercise, strengthen physique, promote health, relieve the pressure of life and so on, are gradually paid attention to by people. Especially in the 2008 Beijing Olympic Games success, accelerate the Chinese sports socialization, lifelong physical education thought is widely spread, people's knowledge on sports deepening, the sports population increased substantially, people's sports increasing demand for diversified, high levels of public sports service to put forward higher requirements. How to better meet the needs of people's sports has become an important problem for the city government ${ }^{[1,2]}$.

Modern information technology, represented by Internet of things, cloud computing, mobile Internet and wearable devices, is changing people's life style, and will have an important impact on the sports industry. With the deepening of reform and opening up, the construction and development of public sports has made unprecedented progress ${ }^{[3]}$. It has played an important role in promoting the coordinated development of mass sports, competitive sports and sports industry. However, the current public sports service still exists the backwardness of management system, service ability is not strong, lack of integration of resources and other issues, and cannot meet the growing diversification, multi-level sports demand. The public sports information platform is driving our sports and health industry to a new step with a strong vitality.

\section{Related work}

Modern society is the survival of the information resources, material and energy are of equal importance, due to the use of modern computer technology, methods and contents of information management has brought great changes, information management has become the indispensable function in the organization. Management information system is an important part of information management $^{[4,5]}$. The application and development for many years, the management information system has become a comprehensive, marginal and cross discipline, including communication science, computer science and information science, mathematics, system science and management science. The application of management information system in China began in 1980s. With the rapid development of system integration technology, management information system is developing in the direction of network, intelligence and distribution. The research work of sports information in 
China started in 1950s. The construction of sports information system is to set up the early sports information database, the sports information database is built according to different application requirements of different types and different scales, such as, the State Sports General Administration established "international mass sports information database" and for the games, athletes and other aspects of the database resources. With the rise and development of the Internet, the provincial sports administrative departments pay more attention to the construction of sports information system, have established the sports administration and sports public information website, network office system, in order to make full use of cyber source, the new system with the original database construction integrated together. The construction and application of websites and systems have greatly improved the efficiency of sports management and the application level of the national sports system informatization. The new advanced information collection and delivery mode not only greatly improves the work efficiency, but also greatly improves the informatization level of China's competitive sports management.

\section{Requirement analysis of public sports information management system}

Design and analysis for the system function, combined with the demand of the main analysis, focusing on the effectiveness of the system function, and make the construction of public sports organization effectiveness, effectiveness analysis on system management functions. With regard to the optimization design of functional modules, combined with the main introduction of facilities, we focus on the effective design of resource services and sports news modules. Sports news mainly releases news of different sports events in a timely manner, and the facilities mainly introduce the sports clubs, the service process of the resources, and the introduction of the public sports facilities. Public sports fitness is often related to the provision of sports knowledge, pay attention to the construction of sports classes. Public sports competitions are often combined with the application of organizational construction and organizational modules to realize the planning of relevant activities related to public sports activities. For public sports competitions, combined with different competition rules, in the activities of the registration process, not only can be combined with the form of mobile phones to achieve registration, but also can help the computer to complete the registration. The actual application on the public sports forum, not only for public sports activities, but also do the organizational effectiveness of public sports and the construction of comprehensive scientific management to realize the sports, and pay more attention to the effectiveness of the system user roles. System management is often to achieve system data maintenance, maintenance of system logs, and timely allocation of various roles in the system, and do a good job of stable operation of the system.

\section{Database of public sports management system based on Access}

The design of a public sports management system based on Access database is often guaranteed to have a reasonable system operation framework. The implementation of system stability is mainly based on the B/S model, and do a good job of different levels of analysis. The design of the system module, combined with ASP scripting language, display the results, and do a good job of component implementation, and strengthen the data layer of the effective interaction process. The implementation of database interaction process, combined with the basic form of database connection, do a good job of effective information calling, combined with the effectiveness of sports information management and analysis, to achieve the effectiveness of the system ${ }^{[6]}$. The basic design of the overall architecture of the system is shown in figure 1. 


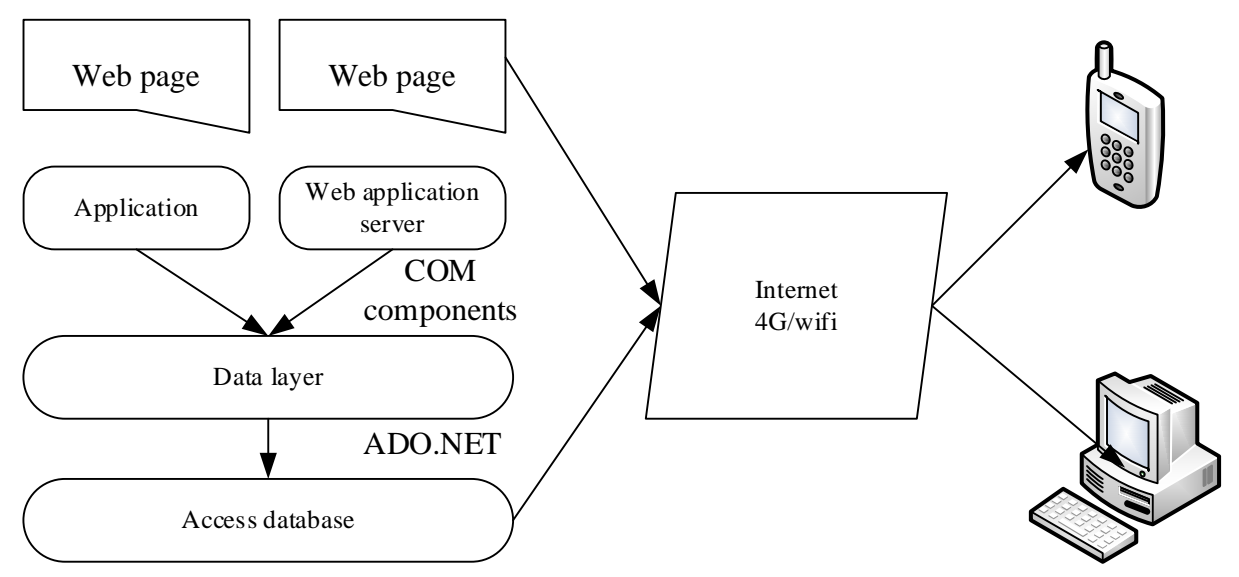

Fig. 1. Database of public sports management system based on Access

\section{System architecture design}

Considering the complexity and particularity of the system, the multi-layer system based on B/S is designed to design the management information system of competitive sports. We use open, easy to use $\mathrm{B} / \mathrm{S}$ mode, can better ensure the decentralized collection, centralized management, resource sharing, unified use of sports management information, while the layered architecture can provide system flexibility, easy to expand and maintain unity ${ }^{[7]}$. The overall structure of the system is shown in figure 2.

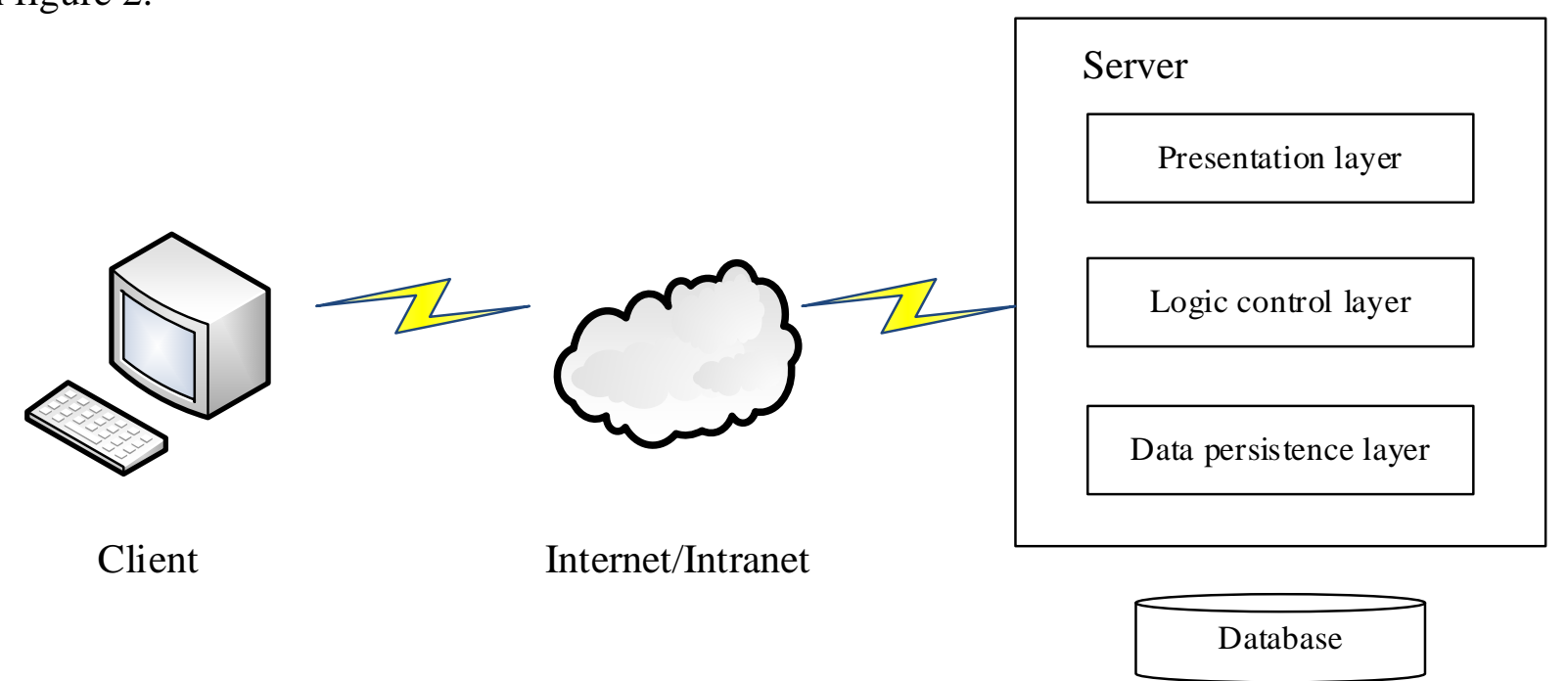

Fig. 2. System architecture based on B/S

The client uses the browser to access the server through the Internet/Intranet. The server can be layered, divided into presentation layer, logical control layer and data persistence layer. These three layers can run either on one server or in the case of larger amounts of data, running separately on the Web server, the application server and the database server. The application server and the Web server are completely separated, because of their own based on computer hardware is separated, the performance which is conducive to give full play to their respective servers; as separate application server and Web server to realize their business functions, application server is responsible for the specific business logic itself, while the Web server is not involved in the core business involves only control logic, which will be conducive to improve the performance of the whole application system, clear and systematic control and maintenance but also conducive to the level of computer function. 


\section{Design of public sports information processing system}

Public sports information processing management system is an intelligent office platform, is a professional sports resource management software. The purpose of developing this software is to collect and transmit data about operation and maintenance of resources and monitor the operation and maintenance of the resources in real time. This software helps to quickly adjust and organize their own management, the fastest and most effective way to meet the needs of partners, with the fastest speed to meet the needs of the consumer; helps to reduce management costs, improve the utilization of equipment resources, can effectively revitalize the tangible assets to sports. The final realization of assist to improve the management of the operation, management, decision-making level and efficiency.

The public sports information processing management system is a Web based application software based on the two major business processes of property management and management. The software provides the management support and decision service for the management of the public sports information system operation and maintenance center. The public sports information management system is a typical enterprise application program based on B/S technology. Its construction needs to take into account the system maintainability, scalability, simple deployment and many other factors. Therefore, the idea of "divide and conquer" is used to divide the problem into several parts and solve them separately, which is beneficial to control, extend and allocate resources.

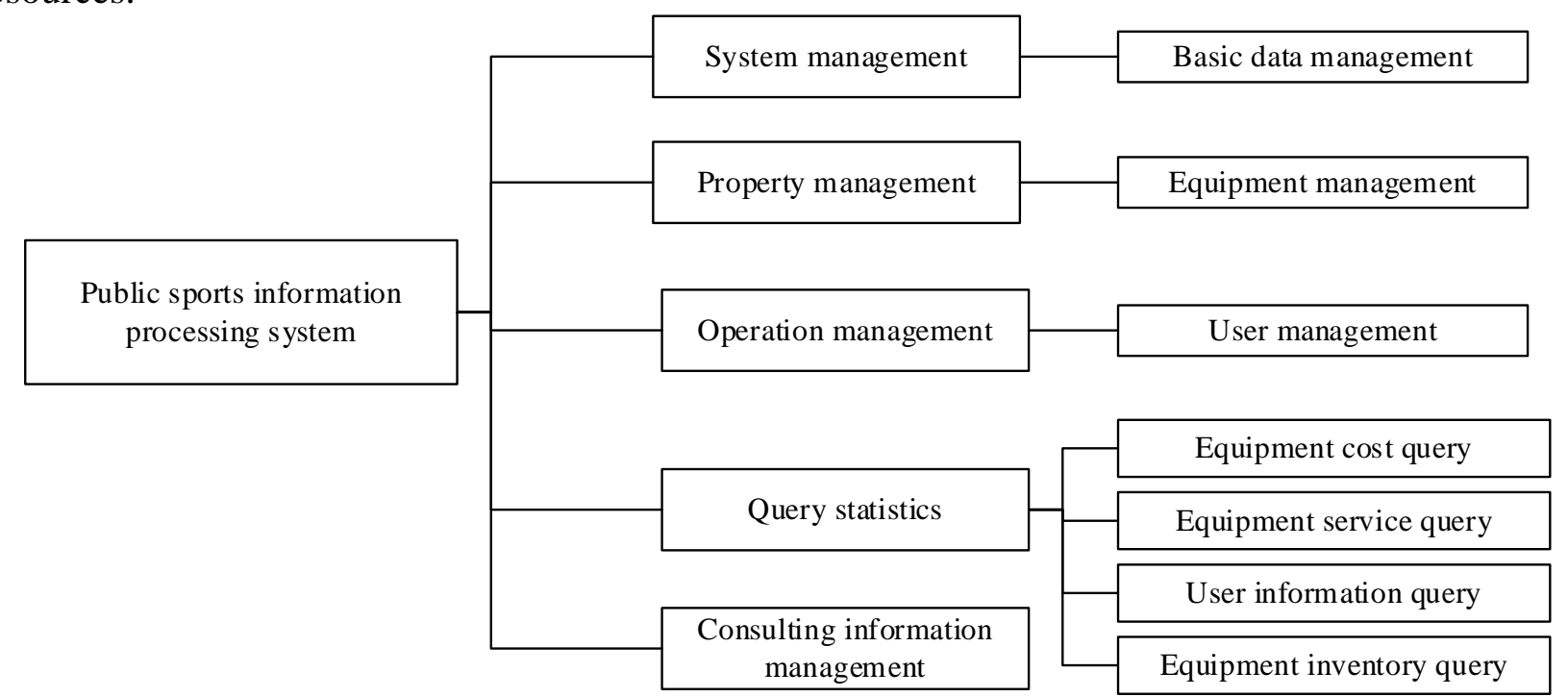

Fig. 3. Public sports information processing system

The public sports information management system is an application software based on Web, which provides corresponding services and information display for sports resource management. The system mainly includes 5 major functional modules, namely, system management module, property management module, management module, query and statistics module, and external information management module, as shown in figure 3.

\section{Conclusion}

In the era of rapid development of information technology, how to make management effective, efficient, effective and management means informatization is an inevitable trend. Needless to say, the power of computers and network technology has been deeply recognized. Effectively and effectively introduce computer management into the operation and maintenance management of stadiums and gymnasiums, which can greatly reduce management costs, improve the efficiency of venue management, and make management more scientific and standardized. Design of public sports management information system based on B/S architecture and Acess database, combined with the network in the form of documentary public sports activities, a comprehensive understanding of the situation of sports facilities, not only the public sports development process to 
achieve better, combined with different intelligence collection, further sustainable development of mobile phone system, more in debugging application process. To achieve better utilization and analysis. Therefore, the design of public sports management information system based on Acess database and $\mathrm{B} / \mathrm{S}$ can be fully promoted and applied to realize the information management of public sports.

\section{References}

[1] Casey Meghan M, Payne Warren R, Eime Rochelle M. Partnership and capacity-building strategies in community sports and recreation programs [J]. Managing Leisure, 2009, 14(3): 167-176.

[2] Kelly Bridget, King Lesley, Bauman Adrian E, Baur Louise A, Macniven Rona, Chapman Kathy, Smith Ben J. Identifying important and feasible policies and actions for health at community sports clubs: A consensus-generating approach [J]. Journal of Science and Medicine in Sport, 2014, 17(1): 61-66.

[3] Zhai Jun, Zhou Kaitao. Semantic retrieval for sports information based on ontology and SPARQL; proceedings of the Information Science and Management Engineering (ISME), 2010 International Conference of, F, 2010 [C]. IEEE.

[4] Scholl Hans J, Carlson Timothy S. Professional sports teams on the Web: a comparative study employing the information management perspective [J]. European Sport Management Quarterly, 2012, 12(2): 137-160.

[5] Caverlee James, Liu Ling, Webb Steve. The SocialTrust framework for trusted social information management: Architecture and algorithms [J]. Information Sciences, 2010, 180(1): 95-112.

[6] Holt Carson, Yandell Mark. MAKER2: an annotation pipeline and genome-database management tool for second-generation genome projects [J]. BMC bioinformatics, 2011, 12(1): 491.

[7] Cao Yan, Yang Lina, Yang Yanli. Three-Layer EPR Management System Architecture and Development Based on B/S Mode; proceedings of the Fourth International Symposium on Information Science and Engineering, F, 2012 [C]. 